\title{
Prevalência da amamentação em crianças menores de dois anos vacinadas nos centros de saúde escola
}

\section{Breastfeeding prevalence among children less than two years old immunized in primary health care school services}

Daniella Fernandes CAMILO ${ }^{1}$

Roberta Villas Boas CARVALHO ${ }^{1}$

Eduardo Freire de OLIVEIRA ${ }^{2}$

Erly Catarina de MOURA ${ }^{3}$

RE S U M O

\section{Objetivo}

Neste trabalho foi desenvolvido estudo transversal com o objetivo de verificar a prevalência da amamentação em 1708 crianças menores de dois anos em Campinas, São Paulo.

\section{Métodos}

As informações sobre idade, sexo, serviço de saúde utilizado e alimentação foram obtidas por entrevistas com mães ou responsáveis durante a Campanha de M ultivacinação de 2001. A amamentação foi classificada como exclusiva, predominante (incluindo outros líquidos, exceto leites), complementar (incluindo outros alimentos e/ou leites) e total (soma de exclusivo, predominante e complementar).

\section{Resultados}

A análise demonstrou que a mediana de amamentação exclusiva foi de 68 dias e a de amamentação total foi de 6,4 meses. No primeiro semestre de vida, $38,1 \%$ das crianças estavam em amamentação exclusiva; $23,0 \%$ em predominante e $14,9 \%$ em complementar. No segundo semestre, $36,5 \%$ das crianças recebiam leite materno; no terceiro $26,4 \%$ e no quarto $13,9 \%$. Crianças usuárias dos serviços públicos e das unidades locais de saúde apresentaram menor risco de desmame do que as usuárias dos serviços privados e de serviços não locais $(p<0,005)$.

\footnotetext{
${ }^{1}$ Acadêmicas, Faculdade de Nutrição, Centro de Ciências da Vida, PUC-Campinas, Brasil.

${ }^{2}$ Acadêmico, Faculdade de Medicina, Centro de Ciências da Vida, PUC-Campinas, Brasil.

${ }^{3}$ Núcleo Pesquisas Epidemiológicas em Nutrição e Saúde (NUPEN S), Faculdade de Saúde Pública, Universidade de São Paulo. Av. Dr. Arnaldo, 715, 01246-904, São Paulo, SP, Brasil. Correspondência para/Correspondence to: E.C. MOURA, Brasil. E-mail: erlycm@usp.br
} 


\section{Conclusão}

Salienta-se a necessidade de estabelecimento de metas visando à amamentação exclusiva até seis meses de idade e total até dois anos ou mais, conforme recomendação da Organização M undial da Saúde e do M inistério da Saúde.

Termos de indexação: aleitamento materno, cuidados primários de saúde, saúde infantil.

\section{A B S T R A C T}

\section{Objective}

A cross-sectional survey was developed aiming to identify the prevalence of breastfeeding among children aged 0-2 years living in Campinas, Sao Paulo state, Brazil.

\section{Methods}

Data on age, sex, health care service used and feeding were obtained by interviewing the mothers or caregivers of 1,708 children during the National Immunization Campaign in 2001. Breastfeeding was classified as exclusive, predominant (including others liquids, except milks), complementary (including others foods and/or others milks) and total (sum of exclusive, predominant and complementary).

\section{Results}

The median exclusive breastfeeding was 68 days and median total breastfeeding was 6.4 months. In the first semester of life, $38.1 \%$ of the children were exclusively breastfed, 23.0 predominantly and $14.9 \%$ complementary breastfed. In the second semester, $36.5 \%$ of the children were breastfed, in the third $26.4 \%$ and in the fourth semester $13.9 \%$. Children who used public and local health care services were less likely to be weaned than the users of private and non-local health care services $(p<0.005)$.

\section{Conclusion}

This article emphasizes the need to establish goals for exclusive breastfeeding up to 6 months of age and for total breastfeeding up to 2 years of age or more, according to the recommendations of the World Health Organization and the Brazilian M inistry of Health.

Index terms: breast feeding, primary health care, child welfare.

\section{N T R O D U ÇÃ O}

O leite materno, como já amplamente descrito na literatura, contém todos os nutrientes essenciais ao crescimento e desenvolvimento da criança, devendo ser 0 alimento exclusivo até os seis primeiros meses da vida ${ }^{1}$. Os benefícios do leite humano são inúmeros, podendo ser destacados o baixo custo, amenorréia materna pós-parto, redução dos níveis de colesterol sérico na vida adulta, menor probabilidade de obesidade, proteção contra infecções, menor chance de alergia e relação afetiva mais intensa entre mãe e filho ${ }^{1-5}$.
A política de saúde da criança no Brasil baseia-se na promoção, na proteção e no apoio ao aleitamento materno, visando à diminuição da mortalidade infantil e à melhoria da saúde. 0 país tornou-se modelo nessa área, implementando, na década de 80 , um programa nacional de promoção à amamentação, contando com o apoio do Ministério da Saúde, da Fundo das Nações Unidas para a Infância (UNICEF) e da Organização M undial da Saúde (OMS S) $)^{6.8}$. Essa abertura trouxe inúmeros avanços, embora ainda hoje seja necessário tratar esse tema como de extrema importância, colocando-o como uma das peças fundamentais dos cuidados primários da saúde ${ }^{9}$. 
A té a década de 80 , não existia no país nenhum programa do governo ou organização responsável pela elaboração e coordenação das atividades relacionadas ao aleitamento. Em 1981, foi criado o Programa Nacional de Incentivo ao Aleitamento $M$ aterno (PNIAM), ligado ao antigo Instituto Nacional de Alimentação e Nutrição. Já na década de 90, na Reunião de Cúpula M undial em Favor da Infância, o Brasil assumiu o compromisso de reduzir a mortalidade infantil no país, utilizando a prática do aleitamento materno como uma de suas principais armas ${ }^{6}$.

Em 1999, durante a segunda etapa da Campanha Nacional de Vacinação contra a Paralisia Infantil, foi realizado o primeiro estudo urbano sobre amamentação no país, levantando dados sobre a prevalência do aleitamento materno em 25 capitais brasileiras e no Distrito Federal. A mediana de amamentação exclusiva foi de 33 dias, valor muito inferior à recomendação de 180 dias. Esse estudo mostrou claramente a diferença das taxas de aleitamento entre as regiões de nosso país, com uma tendência de crescimento da prática de amamentação no Brasil, comparado com pesquisas anteriores, cuja mediana foi de 7 meses $^{10}$.

São indiscutíveis os avanços da prática do aleitamento materno em nosso país, mas pesquisa como a de 199910,11 ainda demonstra, com clareza, as diferenças regionais e estaduais, indicando a necessidade de novos estudos e programas de promoção adequados para cada comunidade. Por outro lado, estudos para diagnóstico rápido sobre amamentação têm sido indicados em campanhas de vacinação pela maior praticidade, baixo custo e confiabilidade ${ }^{12}$.

Nesse sentido, este estudo teve como objetivo verificar a prevalência do aleitamento materno em menores de dois anos de idade, que compareceram à primeira fase da Campólio, em 2001, nos centros de saúde-escola da região Noroeste de Campinas, São Paulo, a fim de subsidiar ações locais de promoção da amamentação.

\section{CASUÍSTICA M ḾTODOS}

A cidade de Campinas situa-se a 100 quilômetros ao Noroeste da cidade de São Paulo, no Estado de São Paulo. Para fins administrativos e assistenciais, Campinas divide-se em 5 Regiões: Norte, Sul, Leste, Sudoeste e Noroeste. A Região Noroeste comporta 7 unidades básicas de saúde, sendo 3 em estreita parceria com a universidade local, a qual assume responsabilidade assistencial e, principalmente, de formação de recursos humanos.

Após avaliação e aprovação em Comitê de Ética da Universidade, foi realizado este estudo transversal nos Centros de Saúde Escola Integração, Ipaussurama e Campos Elíseos, durante a primeira fase da Campanha Nacional de Multivacinação de 2001. Dados sobre a data de nascimento, sexo, serviço de saúde utilizado e alimentação, nas últimas 24 horas, das crianças menores de 2 anos de idade, foram coletados em entrevistas com as mães ou responsáveis pelas crianças, pelos acadêmicos dos cursos de M edicina e Nutrição especialmente treinados e supervisionados. Questionou-se também o serviço de saúde rotineiramente utilizado pela criança em estudo, classificando-o como público (serviços municipais, universitários e conveniados ao Sistema Único de Saúde) ou privado (convênios de saúde e particular).

A amamentação foi classificada como exclusiva, predominante, complementar ou nenhuma, conforme recomendação da $\mathrm{OMS} \mathrm{S}^{13}$. Considerou-se amamentação total quando a criança recebia leite materno de forma exclusiva, predominante ou complementar.

A análise dos dados obtidos foi feita pelo programa Statistical Package for Social Sciences (SPSS). Assim, foram calculadas a idade, as medianas e prevalências de amamentação, bem como o risco de desmame conforme o sexo e serviço de saúde utilizado, considerando-se nível de significância de $5 \%$ e intervalo de confiança de $95 \%$. 


\section{RES ULTA D O S}

Foram entrevistados 1708 responsáveis por crianças menores de 2 anos de idade. Em 13 casos não foi possível obter todas as informações: 3 pessoas desconheciam completamente a alimentação recebida pela criança, outras 3 se ela recebia outro líquido além do leite artificial, 3 se ela recebia outros alimentos além do leite artificial e 3 qual o serviço de saúde mais utilizado pela criança, o que impediu a classificação do tipo de amamentação em apenas 3 casos.

Das entrevistas realizadas, $78,7 \%$ foram respondidas pela mãe da criança; $10,6 \%$ pelo pai; $8,4 \%$ por outros parentes e $2,3 \%$ por outras pessoas. Do total, $47,5 \%$ das crianças eram do sexo feminino e $52,5 \%$ do sexo masculino. Cerca de $58 \%$ eram usuárias de serviços públicos e $42,1 \%$ de serviços privados.

A mediana de amamentação exclusiva da população estudada foi de 68 dias e a mediana de amamentação total foi de 6,4 meses, sendo respectivamente 70 dias e 4,8 meses no Centro de Saúde Integração, 76 dias e 7,3 meses no Ipaussurama e 60 dias e 6,5 meses no Campos Elíseos.

A prevalência de aleitamento materno exclusivo no primeiro mês de vida foi de $69,2 \%$ e de $92,3 \%$ para aleitamento total. No primeiro semestre de vida, $38,1 \%$ das crianças estavam em amamentação exclusiva; $23,0 \%$ em amamentação predominante; $14,9 \%$ em complementar, totalizando $76,0 \%$ das crianças com leite materno e $24,0 \%$ não receberam-no. No segundo semestre, apenas $36,5 \%$ das crianças recebiam leite do peito; no terceiro semestre $26,4 \%$ e no quarto semestre $13,9 \%$ (Tabela 1 ). Ao final do primeiro ano de vida, apenas $33,3 \%$ das crianças recebiam leite materno e $7,1 \%$ ao final do segundo ano. A prevalência de aleitamento materno no primeiro ano de vida foi de $54,9 \%$ no segundo foi de $20,8 \%$.

Na evolução do tipo de amamentação (Figura 1), observa-se, de um modo geral, uma queda drástica do aleitamento exclusivo nos primeiros meses de vida, passando abruptamente de $38 \%$ no quarto mês a $14 \%$ no quinto e $8 \%$ no sexto mês. Por outro lado, o desmame também aumenta gradativamente até $o$ final do primeiro ano de vida, quando assume uma certa estabilidade até quase o final do segundo ano.

Não se encontrou diferença quanto à prevalência de amamentação total entre os sexos. Todavia, 42,4\% dos usuários de serviços públicos amamentaram contra $33,5 \%$ dos usuários dos serviços privados $(p=0,0003)$, gerando para estes últimos um risco de desmame $46,0 \%$ acima do risco dos primeiros $(O R=1,46 ; 1,19<\mid C<1,79)$. Os dados também mostraram a aderência ao serviço local de saúde como fator de proteção ao desmame, assim, 42,7\% dos usuários dos centros de saúde em estudo amamentaram contra 35,2\% dos não usuários $(p=0,002)$, representando um risco de desmame $37,0 \%$ maior para estesúltimos do que para os anteriores $(O R=1,37$; $1,12<\mid C<1,68)$.

Tabela 1. Prevalência da amamentação segundo a idade, Campinas, 2001.

\begin{tabular}{lcccc}
\hline \multirow{2}{*}{ Idade (meses) } & \multicolumn{4}{c}{ Prevalência de Amamentação (\%) } \\
\cline { 2 - 5 } & Exclusiva & Predominante & Complementar & Total \\
\hline 0 а 6 & 38,1 & 23,0 & 14,9 & 76,0 \\
6 a 12 & 2,1 & 1,2 & 33,2 & 36,5 \\
12 a 18 & 1,1 & 2,3 & 23,0 & 26,4 \\
18 a 24 & - & 1,1 & 12,8 & 13,9 \\
\hline
\end{tabular}




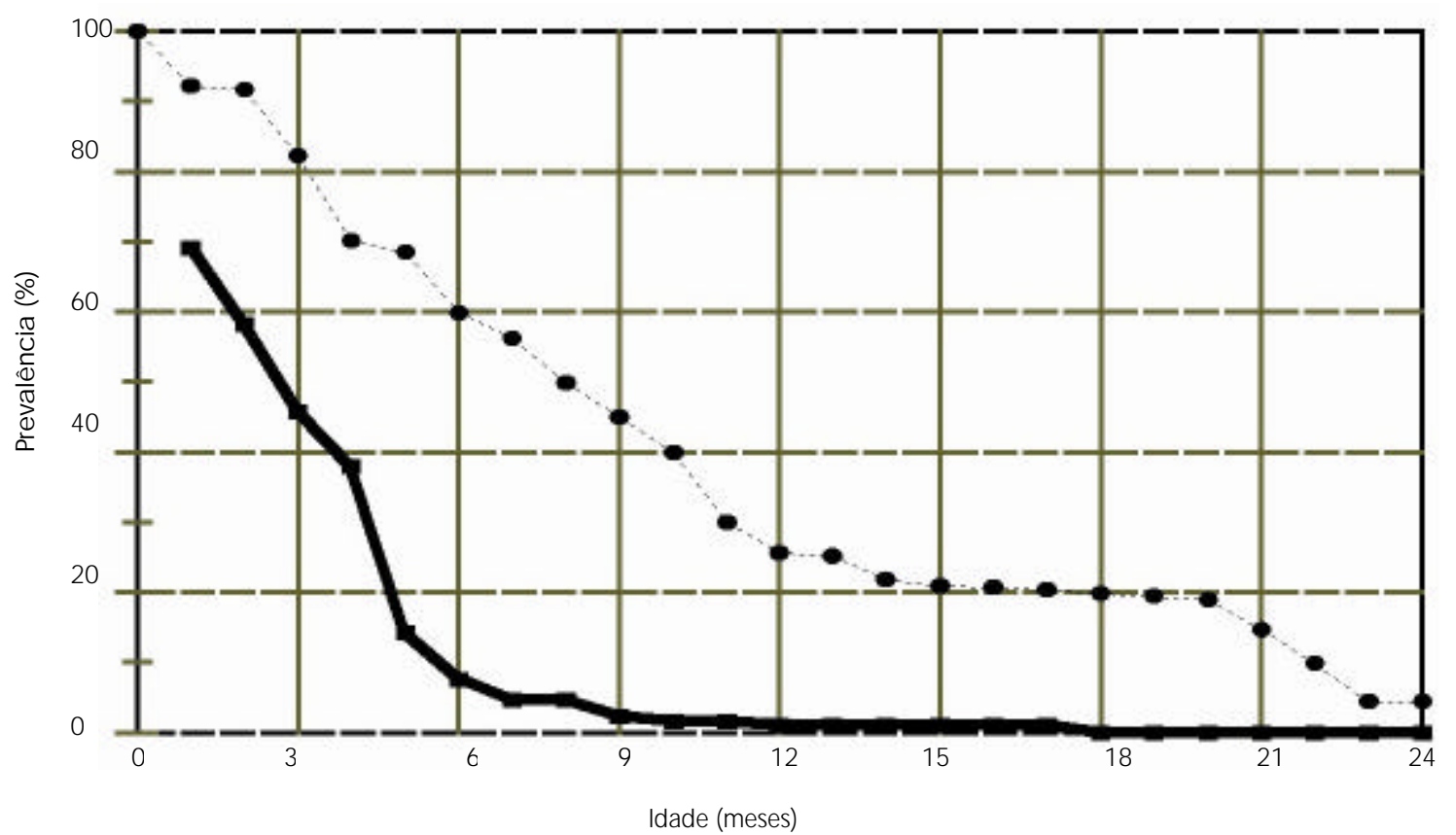

$\rightarrow$ Exclusiva $\quad \ldots *$ Total

Figura 1. Prevalência de amamentação em menores de 2 anos de idade, Campinas, 2001.

\section{I S C U S S Ã O}

A cobertura vacinal na população residente menor de 2 anos de idade foi de $88,0 \%$, de modo que este estudo alcançou cerca de $75,0 \%$ da população estimada residente, conforme dados do último recenseamento, na área de cobertura dos centros de saúde investigados. A menor cobertura vacinal foi a do Centro de Saúde Ipaussurama (63,6\% ); o Centro de Saúde Campos Elíseos apresentou cobertura vacinal de 91,0\% na faixa etária estudada e o Centro de Saúde Integração mais de $100,0 \%$, conforme dados fornecidos pelo Distrito de Saúde Noroeste de Campinas.

Levantamentos de dados deste tipo depende da memória do entrevistado quanto aos alimentos presentes na dieta infantil, se configurando num dos limites deste estudo, embora Monteiro et al. ${ }^{12}$ destaquem a confiabilidade como uma das características principais desta metodologia. Cumpre salientar que a grande maioria das informações foi obtida diretamente da mãe $(78,7 \%)$, que na cultura brasileira é considerada a pessoa mais próxima da criança e responsável pelo seu cuidado.

A mediana de aleitamento materno exclusivo de 68 dias encontrada nessa população, embora muito distante da recomendação oficial de 180 dias, foi o dobro da mediana nacional, que é de apenas 33 dias. Todavia, a mediana de amamentação total de 6,4 meses ficou 8,5\% abaixo da mediana nacional, que é igual a 7 meses e muito distante da recomendação oficial de 24 meses ou mais ${ }^{10}$.

A mediana de aleitamento materno exclusivo em Campinas (68 dias ou 2,2 meses) foi maior do que os valores apontados, na última década, em localidades como João Pessoa, PB, onde o valor encontrado foi 16,5 dias ${ }^{14}$; em Ouro Preto, MG, 17 dias ${ }^{15}$; em Botucatu, SP, 17 dias ${ }^{16}$; em Montes Claros, MG, 27 dias ${ }^{17}$; em Florianópolis, SC, 53 dias $^{14}$; em Piracicaba, SP variou de 3 a 30 dias, dependendo da comunidade 
rural estudada ${ }^{18}$; em São Paulo, SP, a mediana foi de 10 dias junto às crianças de mães trabalhadoras ${ }^{19}$. Todavia, a mediana de aleitamento total (195 dias ou 6,4 meses) foi menor do que a de 238 dias identificada em Florianópolis ${ }^{14}$, de 8,7 meses em M ontes Claros $^{17}$ e de 9 meses em Feira de Santana, BA ${ }^{20}$; similar aos dados de Alterosa, MG, que apontam 6,3 meses ${ }^{21}$ e em João Pessoa, 195 dias $^{14}$; e maior do que a mediana de 71 dias em Ouro Preto ${ }^{15}, 4$ meses em Pelotas, RS'22, 5 meses em São Carlos, $\mathrm{SP}^{23}, 167$ dias em Botucatu ${ }^{16}$ e 150 dias entre as crianças de mães trabalhadoras de São Paulo ${ }^{19}$.

Ao analisar a prevalência do aleitamento, observa-se nos primeiros 30 dias que a prevalência de amamentação exclusiva $(69,2 \%)$ e total $(92,3 \%)$ é alta, quando comparada a outras localidades brasileiras, como exemplo, São Carlos $^{23}$, que apresentou $52,4 \%$ das crianças menores de 1 mês em aleitamento exclusivo. De um modo geral, a média brasileira para aleitamento exclusivo no primeiro mês é de $57,8 \%$, variando de 42,2 a $71,8 \%$ na Região Norte, de 42,1 a 80,9\% na Região Nordeste, de 35,9 a 75,7 na Região Centro-Oeste, de 38,8 a $59,4 \%$ na Região Sudeste e de 68,4 a $75,1 \%$ na Região Sul, que apresenta os melhores índices do país ${ }^{10}$. Em São Paulo, Souza et al. ${ }^{24}$ encontraram $42,2 \%$ das crianças menores de 3 meses em amamentação exclusiva, enquanto que este estudo apontou $56,6 \%$ sendo que em São Paulo, $49,8 \%$ das crianças menores de 1 ano recebiam leite materno e em Campinas 55,7\%.

A prevalência de aleitamento materno exclusivo entre os menores de 4 meses $(51,1 \%)$ também foi maior do que a identificada, por Vieira et al. ${ }^{19}$, em Feira de Santana $(45,6 \%)$, inclusive no primeiro semestre de vida quando se encontrou $38,1 \%$ das crianças campineiras em amamentação exclusiva e em Feira de Santana 36,9\%. Porém, a prevalência de aleitamento total nas crianças menores de seis meses foi maior em Feira de Santana $(82,2 \%)$ do que em Campinas (76,0\% ).

De um modo geral, a curva de declínio de amamentação exclusiva e total (Figura 1) é semelhante à de Florianópolis e João Pessoa ${ }^{14}$, Montes Claros $^{17}$ e Ouro Preto ${ }^{15}$, embora a prevalência de aleitamento exclusivo em João Pessoa e Ouro Preto seja menor do que em Campinas.

\section{O N C L U S Ã O}

Os dados obtidos neste estudo quanto à amamentação exclusiva em menores de 4 meses se encontram entre os mais altos (prevalência acima de 40\% ) do Estado de São Paulo25, mas não quanto à amamentação total em menores de 1 ano.

Quanto à mediana de amamentação exclusiva, os dados apresentados neste trabalho ( 2 a 2,5 meses com média igual a 2,2) são maiores do que todas as outras regiões do país, que variam de 0,7 a 1,7 meses $^{10}$. Enquanto que, no país, apenas $13,0 \%$ das crianças eram amamentadas exclusivamente no período entre 4 e 6 meses, neste estudo foi de $21,5 \%$. Isto reflete, nos locais estudados, do trabalho conjunto da Secretaria Municipal da Saúde de Campinas e da PUCCampinas no incentivo à amamentação exclusiva, ainda que distante da recomendação oficial de aleitamento materno exclusivo pela OMS, até 6 meses e total até 2 anos ou mais ${ }^{10}$. Também evidencia o desafio de aumentar este índice, tendo como meta a amamentação exclusiva até 6 meses para todas as crianças.

No entanto, este estímulo parece focado no aleitamento exclusivo, uma vez que a duração do aleitamento total é bem menor do que os dados das Regiões Norte, Centro-Oeste, Sul e Nordeste do país. Enquanto que, no Brasil ${ }^{10}$, $37,0 \%$ das crianças eram amamentadas até o final do primeiro ano de vida e $18,0 \%$ até o final do segundo ano, este estudo encontrou, respectivamente nas mesmas faixas etárias, $26,0 \%$ e $12,0 \%$, isto reflete uma menor duração de aleitamento total, em Campinas, ainda que a introdução de alimentos ocorra mais tardiamente.

Embora estudos como este, e outros nacionais ou internacionais, vêm demonstrando 
um aumento importante na situação da prevalência de aleitamento materno, as metas oficiais de aleitamento materno exclusivo e continuado ainda estão longe de serem atingidas. Assim, considerando ações de re-organização da atenção primária em Campinas, com a reciclagem em todas as áreas para os profissionais da saúde, visando à territorialização e focando o atendimento na saúde da família, é fundamental rever a qualidade da assistência à saúde da mulher e da criança. Metas devem ser estabelecidas, buscando não somente a extensão e a ampliação da amamentação exclusiva até 6 meses, mas também do aleitamento total até pelo menos 2 anos de idade, evitando a introdução precoce de alimentos complementares.

\section{RE FERÊ N C IAS}

1. Janke JR. The incidence, benefits and variables associated with breastfeeding: implications for practice. Nurse Pract 1993; 18:22-3, 28, 31-2.

2. Giugliani ERJ. 0 aleitamento materno na prática clínica. J Pediatr 2000; 21:238-52.

3. Victora CG, Vaughan JP, Lombardi C, Fuchs SMC, Gigante LP, Smith PG, et al. Evidence for protection by breast-feeding against infant deaths from infectious diseases in Brazil. Lancet 1987; 8:319-22

4. Von Kries R, Koletzko B, Sauerwald T, Von Mutius $E$. Does breast-feeding protect against childhood obesity? Adv Exp Med Biol 2000; 478:29-39.

5. Wagner $\mathrm{CL}$, Wagner MT, Hulsey TC. Factors influencing a mother's decision to breastfeed. Adv Exp Med Biol 2000; 478:435-6.

6. Araújo MFM. Situação e perspectivas do aleitamento materno no Brasil. In: Carvalho RM, Tamez RN. Amamentação: bases científicas para a prática profissional. Rio de Janeiro: Guanabara Koogan; 2002. p.1-9.

7. Kummer SC, Giugliani ERJ, Susin LO, Folletto JL, Lermen NR, Wu VYJ, et al. Evolução do padrão de aleitamento materno. Rev Saúde Pública 2000; 34:143-8
8. Réa M F, Berquó ES. Impact of the Brazilian national breast-feeding programme on mothers in Greater São Paulo. Bull World Health Organ 1990; 68:365-71.

9. Sanghvi TG. Melhora da eficácia em função dos custos da promoção do aleitamento materno em maternidades. New York: UNICEF; 1996.

10. Ministério da Saúde (Brasil). Secretaria de Política de Saúde. Organização Pan Americana de Saúde. Guia alimentar para crianças menores de 2 anos. Brasília: Ministério da Saúde; 2002.

11. Lamounier JA. Tendências do aleitamento materno no Brasil. Rev M éd M inas Gerais 1999; 9:59-66.

12. Monteiro $C A$, Venâncio SI, Kitoko P, Réa MF, Silva NN. Diagnóstico rápido de práticas de alimentação infantil em campanhas de vacinação: descrição de uma proposta. Anais do 40 Congresso Brasileiro de Epidemiologia, 1998. p.310.

13. Organização M undial da Saúde. Indicadores para avaliar as prácticas de lactância materna. Genebra; 1992.

14. Kitoko PM , Réa M F, Venâncio SI, Vasconcelos ACCP, Santos EKA, M onteiro CA. Situação do aleitamento materno em duas capitais brasileiras: uma análise comparada. Cad Saúde Pública 2000; 16:1111-9.

15. Passos M C, Lamounier JA, Silva CAM, Freitas SN, Baudson MFR. Práticas de amamentação no município de Ouro Preto, MG, Brasil. Rev Saúde Pública 2000; 34:617-22.

16. Carvalhaes MABL, Parada CMGL, Manoel CM, Venâncio SI. Diagnóstico da situação do aleitamento materno em área urbana do Sudeste do Brasil: utilização de metodologia simplificada. Rev Saúde Pública 1998; 32:430-6.

17. Caldeira AP, Goulart EMA. A situação do aleitamento materno em Montes Claros, M inas Gerais: estudo de uma amostra representativa. J Pediatr 2000; 76: 65-72.

18. Tabai KC, Carvalho JF, Salay E. Aleitamento materno e a prática de desmame em duas comunidades rurais de Piracicaba, SP. Rev Nutr 1998, 11: 173-83. 
19. Réa M F, Venâncio SI, Batista LE, Santos RG, Greiner T. Possibilidades e limitações da amamentação entre mulheres trabalhadoras formais. Rev Saúde Pública, 1997; 31:149-56.

20. Vieira GO, Glisser M, Araújo SPT, Sales AN. Indicadores do aleitamento materno na cidade de Feira de Santana, Bahia. J Pediatr 1998; 74:11-6.

21. Pereira FA, Silva AC, Valentini AS, Carvalho AM, Souza AF, Candotti KL. Fatores relacionados à interrupção precoce do aleitamento materno em Alterosa/MG. Rev Méd Minas Gerais 1999; 9: 5-9.

22. Horta BL, Olinto MTA, Victora CG, Barros FC, Guimarães PRV. Amamentação e padrões alimentares em crianças de duas coortes de base populacional no Sul do Brasil: tendências e diferenciais. Cad Saúde Pública 1996; 12 (Supl): 43-8.

23. Montrone VC, Arantes CIS. Prevalência do aleitamento materno na cidade de São Carlos, São Paulo. J Pediatr 2000; 76:138-42.

24. Souza SB, Szarfarc SC, Souza JM P. Prática alimentar no primeiro ano de vida, em crianças atendidas em centros de saúde escola do município de São Paulo. Rev Nutr 1999; 12:167-74.

25. Venâncio SI, Escuder MML, Kitoko P, Rea MF, Monteiro $C A$. Freqüência e determinantes do aleitamento materno em municípios do Estado de São Paulo. Rev Saúde Pública 2002; 36:313-18.

Recebido para publicação em 3 de julho de 2002 e aceito em 2 de abril de 2003. 